\title{
Arbor
}

\section{¿Patrimonio Único?}

\section{Cesar Antón Beltrán}

Arbor CLXXX, 710 (Febrero 2005), 363-369 pp.

La Comunidad de Castilla y León recibió las transferencias sanitarias en un escenario diferente al que se habían producido con anterioridad. Los elementos que determinaban estas diferencias, sin ánimo de ser exhaustivos eran los siguientes:

- Por primera vez las transferencias iban ligadas a un nuevo modelo de financiación que se presumía estable, cuando hasta la fecha los modelos previos se planteaban dentro de un escenario temporal.

- El nuevo modelo de financiación integraba la financiación sanitaria dentro de la financiación global de la Comunidad Autónoma.

- Se realizaban transferencias simultáneamente a diez Autonomías.

- En la práctica las transferencias suponían la desaparición del INSALUD.

Este conjunto de situaciones planteaba una serie de incertidumbres de cara al futuro, que podían resumirse en dos:

- Suficiencia del nuevo modelo de financiación

- La forma en que se establecerían las relaciones entre Comunidades Autónomas y entre estas y el Ministerio de Sanidad y Consumo en un modelo sanitario descentralizado.

Es cierto que las transferencias sanitarias suponían además oportunidades para Castilla y León. Gestionar servicios públicos en un ámbito descentralizado permite dar respuestas específicas a necesidades concretas de la población a la que van dirigidos éstos. 


\section{Cesar Antón Beltrán}

Tradicionalmente la gestión del INSALUD tendía a buscar una homogeneidad entre los distintos territorios en que prestaba asistencia sanitaria. Esta búsqueda de criterios comunes se plasmaba en los diferentes indicadores de gestión que establecía y que a la postre dificultaban dar respuestas diferenciadas. Por otra parte determinado tipo de recursos asistenciales y prestaciones se prestaban con un modelo centralizado, muy eficiente desde el punto de vista de empleo de recursos, pero que obligaba a desplazamientos constantes de los ciudadanos. Este último criterio planteaba diferencias importantes en la dotación de determinadas prestaciones y recursos de los denominados de tercer nivel, referencia o superespecialización en las distintas Comunidades Autónomas.

Pero la realidad de Castilla y León casa mal con un modelo centralizado.

En una extensión de $94.224 \mathrm{Km}^{2}$, la mayor de todas las Comunidades Autónomas, lo cual supone el 18,6 \% de la superficie española, conviven 2.493.918 habitantes (el 5,77 \% de la población existente en España, con datos del Padrón correspondientes a 1 de enero de 2004). Así, mientras la densidad de población media del territorio nacional es de 85 habitantes / $\mathrm{Km}^{2}$, en Castilla y León es 26 habitantes $/ \mathrm{Km}^{2}$. Tres veces inferior.

Además, esta densidad media se reduce a 15 habitantes $/ \mathrm{Km}^{2}$ en el medio rural.

La población reside en 2.248 municipios (el 27,7 \% de los municipios nacionales), de los cuales el $74 \%$ tienen menos de 500 residentes (el 47 \% en España, o el 36 \% si descontamos los correspondientes a Castilla y León).

Es decir, a la baja densidad de población se une la dispersión de ésta en múltiples núcleos, situación que supone dificultades importantes a la hora de prestar una serie de servicio públicos y entre ellos los sanitarios.

Además nuestra región, pese a la fama que tiene respecto a una orografía con escasos relieves - la meseta castellana - se encuentra rodeada por montañas y con un nivel medio de altitud superior al resto del territorio español (31,6\% de su territorio por encima de los 1.000 metros, frente al $18,4 \%$ del territorio nacional), lo cual supone una climatología rigurosa.

El conjunto de estas situaciones enmarca el mayor reto que tiene la sanidad en Castilla y León y que es el de la ACCESIBILIDAD.

Es preciso, y nuestros ciudadanos cada vez lo demandan más, llegar con el mayor número de servicios sanitarios a los lugares en que estos residen. Esta situación conlleva que, en muchos casos, haya que primar la accesibilidad frente a la eficiencia y que el coste de la prestación se incremente. Aún en el caso de poder establecer servicios de referencia para 


\section{¿Patrimonio Único?}

un conjunto de poblaciones es preciso tener en cuenta que el coste del transporte sanitario también es mayor, determinado por las grandes distancias existentes entre núcleos de población y hospitales de referencia y que en bastantes casos superan los 100 kilómetros de distancia.

Por otra parte, la movilidad de muchas personas del medio rural se encuentra limitada, hecho ligado sobre todo al mayor envejecimiento que existe en este medio, que en líneas generales podríamos decir que tiene tasas de envejecimiento dobles a las del medio urbano y por tanto un mayor incremento del grado de dependencia o discapacidad de las personas.

Otra de las características de nuestra región, ya apuntada antes, es la del grado de envejecimiento existente. La edad de la población condiciona en gran parte las necesidades de asistencia sanitaria y por tanto el que exista un mayor envejecimiento relativo de la población en Castilla y León supone la necesidad de incrementar el gasto sanitario. El índice de envejecimiento es superior al $22 \%$ (población de más de 65 años sobre el total de habitantes), con provincias, como Soria, que supera el $26 \%$, o como Zamora, con índices superiores al $27 \%$, en tanto que la media nacional se encuentra en cifras cercanas al $17 \%$.

La influencia del factor envejecimiento en la actividad sanitaria ha sido estudiada por diferentes autores, y supone que la población de mayores de 65 años consume el doble del número de consultas e intervenciones quirúrgicas que los menores de esta edad. En el caso de tener en cuenta los ingresos hospitalarios, el consumo de recursos es más de cuatro veces superior a lo consumido por el grupo de menores de 65 años.

Con el fin de dar respuesta a este tipo de situaciones la Junta de Castilla y León planteó un programa de legislatura basado en 6 líneas estratégicas:

1. Un Sistema Autosuficiente. Con el objetivo común de mejorar la salud colectiva e individual. Traduce la aspiración de ser capaces de proporcionar los servicios sanitarios que nuestra población necesite, dentro de la Comunidad Autónoma.

Las actuaciones integradas en esta línea estratégica tienen que ver con la ampliación de la cartera de servicios con diferentes prestaciones, entre las cuales destacamos las siguientes:

- Atención bucodental, no solo enfocada en el segmento de población infantil. También se plantean programas en colectivos de personas con discapacidad, mayores, y en el embarazo.

- Cuidados Intensivos pediátricos, de los cuales nuestra Comunidad Autónoma carecía y que se implantarán en dos hospitales. 
- Incremento importante en los recursos destinados a los pacientes oncológicos y muy especialmente del número de aceleradores lineales.

- Desarrollo importante de los recursos de asistencia psiquiátrica considerados de tercer nivel (Unidades específicas para trastornos duales, Hospitalización infanto-juvenil, Centros de Día y Unidades de Convalecencia Psiquiátrica).

- Detección precoz de la hipoacusia infantil, programa destinado a todos los recién nacidos de Castilla y León.

Pero la autosuficiencia debe también entenderse desde el punto de vista de las infraestructuras en las cuales se realizan las prestaciones sanitarias. Estamos hablando de los hospitales, centros de salud y consultorios locales.

Todos los hospitales se encuentran o tendrán a lo largo de estos años diferentes fases de construcción o reforma. Se actuará sobre 59 Centros de Salud (32 de ellos de nueva construcción) y se reformarán más de 1.100 Consultorios Locales.

Otro de los retos relacionados con la autosuficiencia es el de atención a la dependencia, planteada como una red asistencial en que se integre progresivamente la atención a las necesidades sanitarias y sociales del ciudadano.

2. La segunda línea, deriva directamente de las características demográficas antes señaladas. Estamos refiriéndonos a la Accesibilidad.

Dentro de esta línea nos referimos a actuaciones encaminadas a amortiguar el efecto pernicioso que tienen las variables sociodemográficas en la forma en que se presta la asistencia sanitaria, como son:

- El fomento de la atención especializada en el medio rural, bien con la dotación de Centros de Especialidades en zonas periféricas o alejadas de los hospitales de referencia de la región (Valle de Laciana, Benavente, Ciudad Rodrigo, Valle del Tietar, Merindades y Montaña Palentina), bien con el desplazamiento de especialistas a determinados Centros de Salud.

- Otra actuación importante es la relacionada con la atención y el transporte sanitario de carácter urgente y la atención a las emergencias. En este sentido se ha planteado la extensión al medio rural de las Unidades Medicalizadas de Emergencias (UMEs), de tal modo que de las 22 existentes en la actualidad, 12 se encuentran afectadas a zonas rurales. Por otra parte se dispone de cuatro he- 


\section{¿Patrimonio Único?}

licópteros medicalizados, que permiten cubrir el territorio de la Comunidad Autónoma en isócronas aceptables.

- La accesibilidad también se contempla desde el punto de vista de facilitar las prestaciones sanitarias en el ámbito temporal. En este sentido quizás la actuación más relevante sea la aprobación de un Plan de Reducción de Listas de Espera, con vigencia hasta el año 2007 y con los siguientes objetivos de demora máxima:

\begin{tabular}{|l|l|l|l|}
\hline \multicolumn{1}{|c|}{ Año } & \multicolumn{1}{|c|}{ L.E. Quirúrgica } & \multicolumn{1}{|c|}{$\begin{array}{c}\text { L.E. Consultas } \\
\text { Externas }\end{array}$} & \multicolumn{1}{c|}{$\begin{array}{c}\text { L.E. Pruebas } \\
\text { Diagnósticas }\end{array}$} \\
\hline 2004 & $<165$ días & $<60$ días & $<30$ días \\
\hline 2005 & $<150$ días & $<45$ días & $<25$ días \\
\hline 2006 & $<130$ días & $<35$ días & $<22$ días \\
\hline 2007 & $<100$ días & $<30$ días & $<20$ días \\
\hline
\end{tabular}

Plan que se verá reforzado en breve con la aprobación de un Decreto que garantice los plazos respecto a la prestación quirúrgica.

- Actuaciones encaminadas a facilitar desde un punto de vista temporal el acceso a otras prestaciones tiene que ver con la ampliación en el funcionamiento en horario de tarde de los Centros de Salud que radiquen en municipios de más de 10.000 habitantes, o la implantación del triage o clasificación por niveles de urgencia en todos los servicios de urgencia de nuestros hospitales.

3. Se busca, dentro de la tercera línea estratégica, un compromiso con la calidad. Los diferentes Planes de Gestión de Área, contemplan actuaciones en este sentido, pero hay que dar un paso más, dentro de lo que sería integrar los aspectos de calidad en el trabajo diario y establecer la mejora continúa como filosofía general de trabajo, estamos hablando de crear una Agencia para la Calidad y Acreditación del Sistema Sanitario.

4. Otra línea fundamental es conseguir una verdadera participación de los profesionales en el sistema, con corresponsabilidad en la toma de decisiones. Aquí las actuaciones tienen que ver el modelo de recursos humanos que se desea para esta Autonomía. Un modelo basado en la mejora de la competencia profesional, con programas de formación continua. Un modelo que estimule la motivación y el compromiso, favoreciendo la estabilidad en el empleo y con el diseño de una carrera profesional. En fin, un modelo que además permita la participación de la sociedad, fomentando las Consejos de Salud de Zona.

5. En el terreno de la Salud Pública, las actuaciones más novedosas tienen que ver con la creación de la Agencia de Protección de la Salud y 
Seguridad Alimentaria. Dando respuesta a una demanda social creciente que tiene que ver con la repercusión que tienen sobre nuestra salud las condiciones del ecosistema. La influencia que sobre éste tiene la población justifica el control y la vigilancia sobre los agentes físicos, químicos y biológicos.

6. El sistema sanitario debe ser además innovador, sobre todo si tenemos en cuenta que es uno de los sectores en que el cambio es una constante. Actuaciones relacionadas con el empleo y difusión en el entorno de trabajo de las tecnologías de la información y telecomunicaciones permiten además dar una respuesta al reto de la accesibilidad, de modo que el médico de familia disponga de la información clínica relevante para la toma de decisiones.

- La informatización de los Centros de Salud, con una aplicación de desarrollo propio, es clave en ese sentido.

- Pero también existen otras actuaciones relacionadas con técnicas Telemédicas, que permiten, bien un acercamiento de las posibilidades diagnósticas, bien la realización de interconsultas entre el Médico de Familia y diferentes especialistas. La Telemedicina tiene su campo esencial en las Zonas Básicas de carácter rural (aproximadamente el 70 \% de las Zonas Básicas de nuestra región).

En las páginas anteriores se han señalado una serie de factores que determinan una respuesta diferenciada a los problemas sanitarios respecto a otras Autonomías, respuesta que se plantea desde las 6 líneas estratégicas que definen las actuaciones en materia sanitaria de la Consejería de Sanidad.

Existe otra serie de factores, no tan evidentes como los antes señalados, que también dejan sentir su influencia en un modelo sanitario descentralizado y que merecen ser detallados, aunque sea sucintamente y desde la experiencia que da la gestión sanitaria, más que desde aspectos objetivables.

Las transferencias dejan sentir, en la gestión de los servicios sanitarios, lo que se ha denominado en llamar «efecto cercanía». Con él se quiere señalar la existencia de una serie de demandas de prestación de determinados servicios, que obviando, en muchos casos, el componente de planificación y gestión se presentan como una decisión de naturaleza estrictamente "política». Este efecto es más patente en Autonomías de carácter pluriprovincial, especialmente cuando existe un peso equiparable entre las distintas provincias o ciudades. 


\section{¿Patrimonio Único?}

Otro efecto, es el que podríamos definir como «mimetismo» con otras Comunidades Autónomas. Castilla y León se encuentra rodeada por nue ve Autonomías, que en el ejercicio de sus competencias sanitarias y de su realidad sociodemográfica, plantean diferentes prestaciones o establecen prioridades diferentes. Existe una cierta tendencia a solicitar prioridades o prestaciones que si bien pueden tener su justificación en una determinada Comunidad Autónoma, no la tengan en otra.

Por último, un modelo sanitario como el nuestro, precisa de un determinado nivel de coordinación, que podemos afirmar está en sus inicios. La coordinación es necesaria para garantizar una serie de prestaciones de carácter superespecializado, que deben prestarse con criterios de referencia para diferentes regiones. La coordinación también es necesaria a la hora de establecer nuevas prestaciones o la política farmacéutica y para su desarrollo sigue siendo importante el papel del Ministerio de Sanidad. Claro está, que las decisiones que puedan tomarse a este nivel tienen un reflejo en el gasto que se efectúa en las Autonomías y por tanto deben conllevar una financiación adecuada, lo demás es «invitar a que pague otro» y en Castilla y León esto ha sucedido más de lo deseable en los últimos tiempos. 